\title{
Momentous CRISPR-enabled developments
}

\author{
The simplicity and powerful capabilities of CRISPR have led to an explosion of genome-editing applications. Their \\ continued development should be nurtured by scientific and legal environments that discourage and penalize \\ irresponsible uses of the technology.
}

C RISPR has made DNA editing broadly accessible. This is largely because of its simplicity, programmability and precision. Unlike older DNA-editing techniques, which rely on intricate protein engineering, the ability of CRISPR to specifically target DNA sequences is enabled by a designable, easy-to-synthesize guideRNA strand. The 'democratization' of genome editing that CRISPR is enabling provides new hopes for patient care across medical disciplines, and facilitates the development of new therapies with unprecedented accuracy of action, especially for diseases with a well-defined genetic cause.

The optimization of CRISPR for DNA editing has broadened its range of applications, improved its efficiency and accuracy, and led to methods that selectively deliver the CRISPR machinery to target tissues. In a proof-of-concept method, included in this issue, Gang Bao and colleagues describe how genome editing by CRISPR can be activated locally via an applied magnetic field. For this, the CRISPR components are delivered within viral vectors that are inactivated in serum (by the complement system). Via complexation of the vectors with magnetic nanoparticles, the researchers observed that an applied magnetic field triggered a local increase in the rate of cellular uptake of the vectors (with various mechanisms likely to be at play), thereby bypassing their serum inactivation. Both local and systemic administration of the vectors in mice led to enhanced transgene expression only in the tissue or organ where the magnetic field was applied.

The power and simplicity of CRISPR is facilitating strategies that require ex vivo insertion of therapeutically relevant genes in cells that are then implanted into the patient (as is the case of chimaeric antigen receptor $\mathrm{T}$ cells for the treatment of blood cancers, and of drug-producing cells acting as depots). The ease of genetically manipulating cells ex vivo is also enabling therapeutic solutions in disease areas that have not traditionally been targeted by cell therapy. Xiaoyang $\mathrm{Wu}$, Ming $\mathrm{Xu}$ and colleagues report, also in this issue, a proofof-concept therapy for cocaine addiction. The researchers genetically modified mouse skin epidermal stem cells to produce

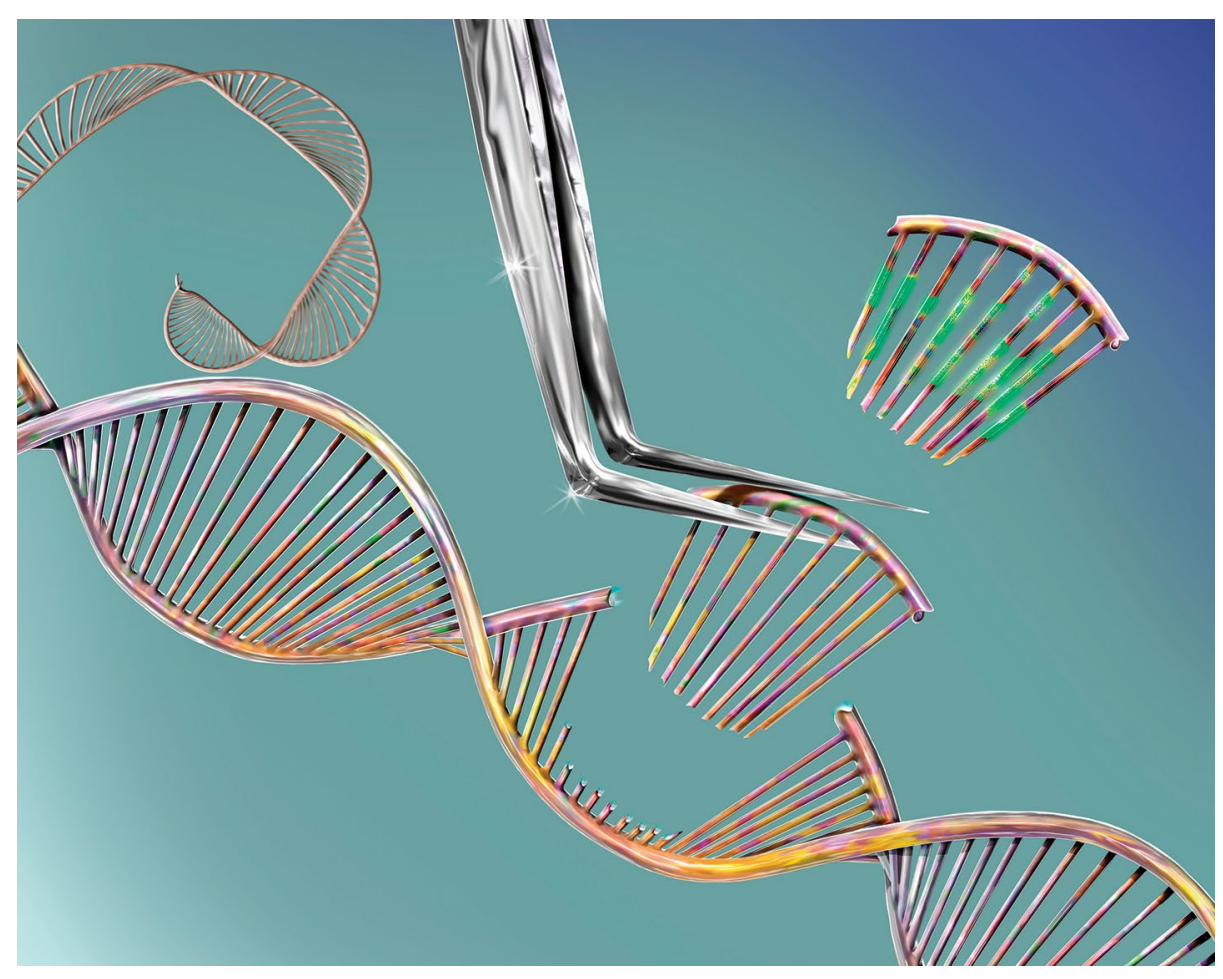

Credit: Keith Chambers/Science Photo Library/Getty Images

an enzyme that hydrolyses cocaine.

The implantation of the genome-edited skin cells in mice led to sustained levels of the enzyme in circulation, which protected the mice from cocaine-seeking behaviour and cocaine overdoses. Although it is unclear whether such a cutaneous gene therapy for cocaine abuse could, on its own, be effective in preventing cocaine overdose in people suffering from drug addiction, the approach may inspire the development of other cutaneous gene therapies.

CRISPR is also enabling fundamental research into disease pathogenesis. For example, high-throughput genetic and phenotypic screens featuring large libraries of mutant cells or mutant organisms have long been used to identify genes involved in dysfunctional processes associated with disease. CRISPR facilitates the assembly of these libraries. A study by Neil Chi, Robert Ross and Adam Engler included in this issue used CRISPR to generate a library of patient-derived isogenic human cardiomyocytes (derived from human pluripotent stem cells) bearing an allelic series of variants in two genes implicated in dilated cardiomyopathy to investigate how compound genetic interactions contribute to the complex heritability of the disease. The researchers found that only cardiomyocytes bearing specific variants of both genes displayed reduced contractility and lessorganized sarcomeres, and confirmed these findings with isogenic mice genetically engineered (also via CRISPR) to carry either or both human gene variants. The in vivo experiments also revealed that haemodynamic stress on the heart mediated the susceptibility of mice carrying both gene variants to dilated cardiomyopathy.

These three studies are but a sample of new biomedical developments enabled by CRISPR. Since the first description of CRISPR as a molecular tool for DNA editing, research into improving the 
efficiency of genome editing has proceeded apace. New uses of the technique for therapy and diagnostics, for uncovering fundamental biology, and for elucidating pathogenesis, are regularly published. Such an explosion of CRISPR-enabled research could, however, be threatened by the very simplicity and powerful capabilities of the technology, which makes it easier for morally questionable experimentation to proceed quickly without proper oversight. As a case in point, He Jiankui's ethically fraught, misguided and botched use of CRISPR to edit human embryos that were allowed to proceed through gestation, first reported in November 2018, has been a wake-up call. The response of the scientific community was swift in its condemnation of this example of reckless experimentation in humans, highlighting the importance of protecting individuals who cannot give consent and of safeguarding the human germline from what is still a largely unproven tool in regard to in vivo applications in humans. Chinese authorities have now confirmed He's claims, including a second pregnancy from a gene-edited embryo, and declared his activities illegal.

In addition to the unknown risks to the babies' health and, potentially, to that of their descendants, He's experiments could hinder future work on CRISPR by shifting public opinion and political and legal stances over the use of CRISPR and the development of genome-editing applications in medicine. To minimize irresponsible uses of the technology (as has been the case with unproven stem-cell therapies), broadly inclusive initiatives should arise on three fronts. First, scientists should accurately and clearly communicate the benefits and perils of the uses of CRISPR to the public, while simultaneously providing assurances that applications are being tested appropriately and carried out with appropriate scientific and ethical oversights. Second, international agreements on the ethical boundaries of the use of genome editing should be reached, and periodically discussed and revised, and these discussions should involve scientific and legal experts, as well as representatives of government, business and all sectors of civil society, as the proposed 'global observatory for gene editing' (Nature 555, 435-437; 2018) is set to achieve. Third, permitted uses of CRISPR in humans and in biological systems of societal importance should be carried out transparently, and their outcomes tracked. Setting up comprehensive training on the ethics of genetic experimentation in humans and on responsible research protocols for it, and the spearheading of inclusive societal debates about the uses of genome editing, will go a long way towards the nurturing of a thriving environment for genome-engineering technology, for the benefit of humankind.

Published online: 7 February 2019 https://doi.org/10.1038/s41551-019-0361-z 